\title{
NEW HOOK-CONTENT FORMULAS FOR STRICT PARTITIONS
}

\author{
GUO-NIU HAN AND HUAN XIONG*
}

\begin{abstract}
We introduce the difference operator for functions defined on strict partitions and prove a polynomiality property for a summation involving the hook length and content statistics. As an application, several new hook-content formulas for strict partitions are derived.
\end{abstract}

\section{INTRODUCTION}

The basic knowledge on partitions, Young tableaux and symmetric functions could be found in [17]. In this paper, we focus on strict partitions. A strict partition is a finite strict decreasing sequence of positive integers $\lambda=\left(\lambda_{1}, \lambda_{2}, \ldots, \lambda_{\ell}\right)$. The integer $|\lambda|=\sum_{1 \leq i \leq \ell} \lambda_{i}$ is called the size of the partition $\lambda$ and $\ell(\lambda)=\ell$ is called the length of $\lambda$. For convenience, let $\lambda_{i}=0$ for $i>l(\lambda)$. A strict partition $\lambda$ can be identified with its shifted Young diagram, which means that the $i$-th row of the usual Young diagram is shifted to the right by $i$ boxes. Therefore the leftmost box in the $i$-th row has coordinate $(i, i+1)$. For the $(i, j)$-box in the shifted Young diagram of the strict partition $\lambda$, we can associate its hook length, denoted by $h_{(i, j)}$, which is the number of boxes exactly to the right, or exactly above, or the box itself, plus $\lambda_{j}$. For example, consider the box $\square=(i, j)=(1,3)$ in the shifted Young diagram of the strict partition $(7,5,4,1)$. There are 1 and 5 boxes above and to the right of the box $\square$ respectively. Since $\lambda_{3}=4$, the hook length of $\square$ is equal to $1+5+1+4=11$, as illustrated in Figure 1 . The content of $\square=(i, j)$ is defined to be $c_{\square}=j-i$, so that the leftmost box in each row has content 1. Also, let $\mathcal{H}(\lambda)$ be the multi-set of hook lengths of boxes and $H_{\lambda}$ be the product of all hook lengths of boxes in $\lambda$.

Our goal is to find some hook-content formulas for strict partitions, by analogy with that for ordinary partitions. For the ordinary partition $\nu$, it is well known that (see $[\underline{3}, \underline{9}, \underline{17}])$

$$
f_{\nu}=\frac{|\nu| !}{H_{\nu}} \quad \text { and } \quad \frac{1}{n !} \sum_{|\nu|=n} f_{\nu}^{2}=1
$$

where $H_{\nu}$ denotes the product of all hook lengths of boxes in $\nu$ and $f_{\nu}$ denotes the number of standard Young tableaux of shape $\nu$. The first author conjectured [4] that

$$
P(n)=\frac{1}{n !} \sum_{|\nu|=n} f_{\nu}^{2} \sum_{\square \in \nu} h_{\square}^{2 k}
$$

2010 Mathematics Subject Classification. 05A15, 05A17, 05A19, 05E05, 05E10, 11P81.

Key words and phrases. strict partition, hook length, content, shifted Young tableau, difference operator.

An extended abstract for this paper appeared in the Proceedings of the FPSAC'16 conference.

* Huan Xiong is the corresponding author. 

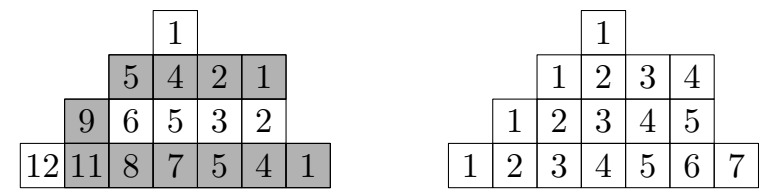

Figure 1. The shifted Young diagram of the strict partition $(7,5,4,1)$ with its hook lengths and contents.

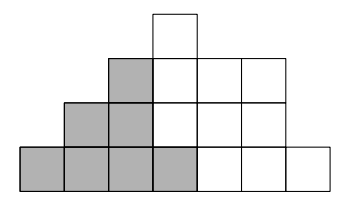

Figure 2. The skew shifted Young diagram of the skew strict partition $(7,5,4,1) /(4,2,1)$.

is always a polynomial in $n$ for all $k \in \mathbb{N}$, which was generalized and proved by Stanley [16], and later generalized in [7] (see also [2, 5, 6, , 8, 10, 11, 12, 13]).

For two strict partitions $\lambda$ and $\mu$, we write $\lambda \supseteq \mu$ if $\lambda_{i} \geq \mu_{i}$ for all $i \geq 1$. In this case, the skew strict partition $\lambda / \mu$ can be identified with its skew shifted Young diagram. For example, the skew strict partition $(7,5,4,1) /(4,2,1)$ is represented by the white boxes in Figure 2, Let $f_{\lambda}$ (resp. $f_{\lambda / \mu}$ ) be the number of standard shifted Young tableaux of shape $\lambda$ (resp. $\lambda / \mu$ ). The following are well-known formulas (see $[1,15,18]$ ) analogous to (1.1):

$$
f_{\lambda}=\frac{|\lambda| !}{H_{\lambda}} \quad \text { and } \quad \frac{1}{n !} \sum_{|\lambda|=n} 2^{n-\ell(\lambda)} f_{\lambda}^{2}=1 .
$$

In this paper, we generalize the latter equality of (1.2) by means of the following results.

Theorem 1.1. Suppose that $Q$ is a given symmetric function, and $\mu$ is a given strict partition. Then

$$
P(n)=\sum_{|\lambda / \mu|=n} \frac{2^{|\lambda|-|\mu|-\ell(\lambda)+\ell(\mu)} f_{\lambda / \mu}}{H_{\lambda}} Q\left(\left(\begin{array}{c}
c_{\square} \\
2
\end{array}\right): \square \in \lambda\right)
$$

is a polynomial in $n$, where $Q\left(\left(\begin{array}{c}c \square \\ 2\end{array}\right): \square \in \lambda\right)$ means that $|\lambda|$ of the variables are substituted by $\left(\begin{array}{c}c \\ 2\end{array}\right)$ for $\square \in \lambda$, and all other variables by 0 .

Theorem 1.2. Suppose that $k$ is a given nonnegative integer. Then

$$
\sum_{|\lambda|=n} \frac{2^{|\lambda|-\ell(\lambda)} f_{\lambda}}{H_{\lambda}} \sum_{\square \in \lambda}\left(\begin{array}{c}
c_{\square}+k-1 \\
2 k
\end{array}\right)=\frac{2^{k}}{(k+1) !}\left(\begin{array}{c}
n \\
k+1
\end{array}\right) .
$$

When $k=0$ we derive the latter identity of (1.2). When $k=1$, Theorem 1.2 becomes

$$
\sum_{|\lambda|=n} \frac{2^{|\lambda|-\ell(\lambda)} f_{\lambda}}{H_{\lambda}} \sum_{\square \in \lambda}\left(\begin{array}{c}
c_{\square} \\
2
\end{array}\right)=\left(\begin{array}{l}
n \\
2
\end{array}\right),
$$


which could also be obtained by setting $\mu=\emptyset$ in the next theorem.

Theorem 1.3. Let $\mu$ be a strict partition. Then

$$
\sum_{|\lambda / \mu|=n} \frac{2^{|\lambda|-\ell(\lambda)-|\mu|+\ell(\mu)} f_{\lambda / \mu} H_{\mu}}{H_{\lambda}}\left(\sum_{\square \in \lambda}\left(\begin{array}{c}
c_{\square} \\
2
\end{array}\right)-\sum_{\square \in \mu}\left(\begin{array}{c}
c_{\square} \\
2
\end{array}\right)\right)=\left(\begin{array}{c}
n \\
2
\end{array}\right)+n|\mu| .
$$

The proofs of those theorems are given in Section 4, by using the difference operator technique.

\section{DiffERENCE OPERATORS}

For each strict partition $\lambda$, the symbol $\lambda^{+}$(resp. $\lambda^{-}$) always represents a strict partition obtained by adding (resp. removing) a box to (resp. from) $\lambda$. In other words, $\left|\lambda^{+} / \lambda\right|=1$ and $\left|\lambda / \lambda^{-}\right|=1$. By analogy with the difference operator for ordinary partitions introduced in [7], we define the difference operator for strict partitions by

$$
D(g(\lambda)):=\sum_{\ell\left(\lambda^{+}\right)>\ell(\lambda)} g\left(\lambda^{+}\right)+2 \sum_{\ell\left(\lambda^{+}\right)=\ell(\lambda)} g\left(\lambda^{+}\right)-g(\lambda),
$$

where $\lambda$ and $\lambda^{+}$are strict partitions and $g$ is a function on strict partitions. Notice that $\#\left\{\lambda^{+}: \ell\left(\lambda^{+}\right)>\ell(\lambda)\right\}=0$ or 1 .

For each skew strict partition $\lambda / \mu$, let ${f^{\prime}}_{\lambda / \mu}:=2^{|\lambda|-|\mu|-\ell(\lambda)+\ell(\mu)} f_{\lambda / \mu}$.

Lemma 2.1. For two different strict partitions $\lambda \supseteq \mu$ we have

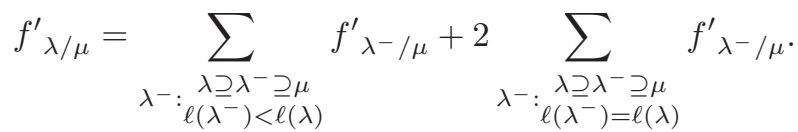

Proof. By the construction of standard shifted Young tableaux we have

$$
f_{\lambda / \mu}=\sum_{\lambda \supseteq \lambda^{-} \supseteq \mu} f_{\lambda^{-} / \mu}
$$

and therefore

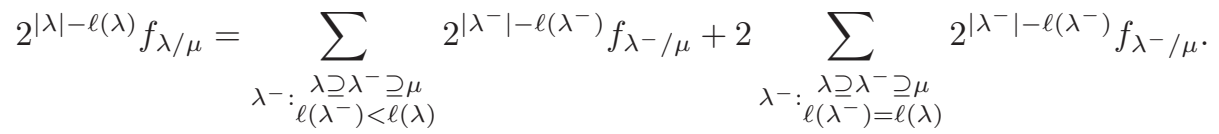

Then by the definition of ${f^{\prime}}_{\lambda / \mu}$ we prove the claim.

Lemma 2.2. For each strict partition $\mu$ and each function $g$ of strict partitions, let

$$
A(n):=\sum_{|\lambda / \mu|=n} f^{\prime}{ }_{\lambda / \mu} g(\lambda)
$$

and

$$
B(n):=\sum_{|\lambda / \mu|=n} f_{\lambda / \mu}^{\prime} D g(\lambda)
$$

Then

$$
A(n)=A(0)+\sum_{k=0}^{n-1} B(k)
$$


Proof. We have

$$
\begin{aligned}
& A(n+1)-A(n)=\sum_{|\gamma / \mu|=n+1} f_{\gamma / \mu}^{\prime} g(\gamma)-\sum_{|\lambda / \mu|=n} f_{\lambda / \mu}^{\prime} g(\lambda)
\end{aligned}
$$

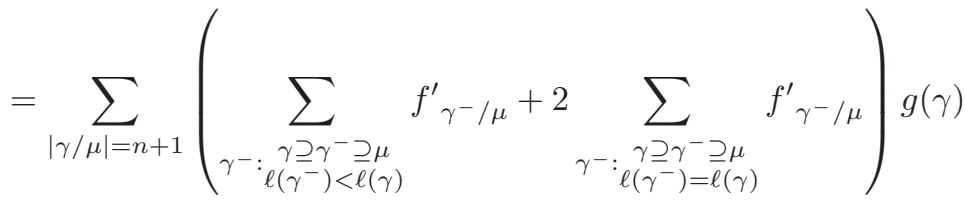

$$
\begin{aligned}
& -\sum_{|\lambda / \mu|=n} f_{\lambda / \mu}^{\prime} g(\lambda) \\
& =\sum_{|\lambda / \mu|=n}{f^{\prime}}_{\lambda / \mu}\left(\sum_{\ell\left(\lambda^{+}\right)>\ell(\lambda)} g\left(\lambda^{+}\right)+2 \sum_{\ell\left(\lambda^{+}\right)=\ell(\lambda)} g\left(\lambda^{+}\right)-g(\lambda)\right) \\
& =\sum_{|\lambda / \mu|=n}{f^{\prime}}_{\lambda / \mu} D g(\lambda)=B(n) \text {. }
\end{aligned}
$$

Thus

$$
\begin{aligned}
A(n+1) & =A(n)+B(n) \\
& =A(n-1)+B(n-1)+B(n) \\
& =\cdots \\
& =A(0)+\sum_{k=0}^{n} B(k) .
\end{aligned}
$$

Theorem 2.3. Let $g$ be a function on strict partitions and $\mu$ be a given strict partition. Then we have

$$
\sum_{|\lambda / \mu|=n} 2^{|\lambda|-|\mu|-\ell(\lambda)+\ell(\mu)} f_{\lambda / \mu} g(\lambda)=\sum_{k=0}^{n}\left(\begin{array}{l}
n \\
k
\end{array}\right) D^{k} g(\mu)
$$

and

$$
D^{n} g(\mu)=\sum_{k=0}^{n}(-1)^{n+k}\left(\begin{array}{l}
n \\
k
\end{array}\right) \sum_{|\lambda / \mu|=k} 2^{|\lambda|-|\mu|-\ell(\lambda)+\ell(\mu)} f_{\lambda / \mu} g(\lambda) .
$$

In particular, if there exists some positive integer $r$ such that $D^{r} g(\lambda)=0$ for every strict partition $\lambda$, then the left-hand side of (2.1) is a polynomial in $n$ with degree at most $r-1$.

Proof. We will prove (2.1) by induction. The case $n=0$ is trivial. Assume that (2.1) is true for some nonnegative integer $n$. Then by Lemma 2.2 we have

$$
\begin{aligned}
\sum_{|\lambda / \mu|=n+1} f_{\lambda / \mu}^{\prime} g(\lambda) & =\sum_{|\lambda / \mu|=n} f_{\lambda / \mu}^{\prime} g(\lambda)+\sum_{|\lambda / \mu|=n} f_{\lambda / \mu}^{\prime} D g(\lambda) \\
& =\sum_{k=0}^{n}\left(\begin{array}{l}
n \\
k
\end{array}\right) D^{k} g(\mu)+\sum_{k=0}^{n}\left(\begin{array}{l}
n \\
k
\end{array}\right) D^{k+1} g(\mu) \\
& =\sum_{k=0}^{n+1}\left(\begin{array}{c}
n+1 \\
k
\end{array}\right) D^{k} g(\mu) .
\end{aligned}
$$


Identity (2.2) follows from the Möbius inversion formula [14].

Example. Let $g(\lambda)=1 / H_{\lambda}$. Then $D g(\lambda)=0$ by Theorem 3.3. The two quantities defined in Lemma 2.2 are:

$$
A(n)=\sum_{|\lambda / \mu|=n} \frac{f_{\lambda / \mu}^{\prime}}{H_{\lambda}} \quad \text { and } \quad B(n)=0 .
$$

Consequently,

$$
\sum_{|\lambda / \mu|=n} \frac{2^{|\lambda|-|\mu|-\ell(\lambda)+\ell(\mu)} f_{\lambda / \mu}}{H_{\lambda}}=\frac{1}{H_{\mu}} .
$$

In particular, $\mu=\emptyset$ implies

$$
\sum_{|\lambda|=n} \frac{2^{|\lambda|-\ell(\lambda)} f_{\lambda}}{H_{\lambda}}=1
$$

or equivalently,

$$
\sum_{|\lambda|=n} 2^{|\lambda|-\ell(\lambda)} f_{\lambda}^{2}=n !
$$

\section{Corners of Strict partitions}

For a strict partition $\lambda$, the outer corners are the boxes which can be removed to get a new strict partition $\lambda^{-}$. Let $\left(\alpha_{1}, \beta_{1}\right), \ldots,\left(\alpha_{m}, \beta_{m}\right)$ be the coordinates of outer corners such that $\alpha_{1}>\alpha_{2}>\cdots>\alpha_{m}$. Let $y_{j}=\beta_{j}-\alpha_{j}$ be the contents of outer corners for $1 \leq j \leq m$. We set $\alpha_{m+1}=0, \beta_{0}=\ell(\lambda)+1$ and call $\left(\alpha_{1}, \beta_{0}\right),\left(\alpha_{2}, \beta_{1}\right), \ldots,\left(\alpha_{m+1}, \beta_{m}\right)$ the inner corners of $\lambda$. Let $x_{i}=\beta_{i}-\alpha_{i+1}$ be the contents of inner corners for $0 \leq i \leq m$ (see Figure 3). The following relations of $x_{i}$ and $y_{j}$ are obvious.

$$
x_{0}=1 \leq y_{1}<x_{1}<y_{2}<x_{2}<\cdots<y_{m}<x_{m} .
$$

Notice that $y_{1}=1$ iff $\lambda_{\ell(\lambda)}=1$.

We define

$$
q_{k}(\lambda):=\sum_{i=0}^{m}\left(\begin{array}{c}
x_{i} \\
2
\end{array}\right)^{k}-\sum_{i=1}^{m}\left(\begin{array}{c}
y_{i} \\
2
\end{array}\right)^{k}
$$

for each $k \geq 0$. For each partition $\nu=\left(\nu_{1}, \nu_{2}, \ldots, \nu_{\ell}\right)$ we define the function $q_{\nu}(\lambda)$ of strict partitions by

$$
q_{\nu}(\lambda):=q_{\nu_{1}}(\lambda) q_{\nu_{2}}(\lambda) \cdots q_{\nu_{\ell}}(\lambda) .
$$

First we consider the difference between the hook length sets of $\lambda$ and $\lambda^{+}=$ $\lambda \cup\{\square\}$ for some box $\square$.

Theorem 3.1. Suppose that $\lambda^{+}=\lambda \cup\{\square\}$ such that $c_{\square}=x_{i}$. If $i=0$, then

$$
\frac{H_{\lambda}}{H_{\lambda^{+}}}=\frac{\prod_{1 \leq j \leq m}\left(\left(\begin{array}{c}
x_{0} \\
2
\end{array}\right)-\left(\begin{array}{c}
y_{j} \\
2
\end{array}\right)\right)}{\prod_{1 \leq j \leq m}\left(\left(\begin{array}{c}
x_{0} \\
2
\end{array}\right)-\left(\begin{array}{c}
x_{j} \\
2
\end{array}\right)\right)} .
$$


If $1 \leq i \leq m$, then

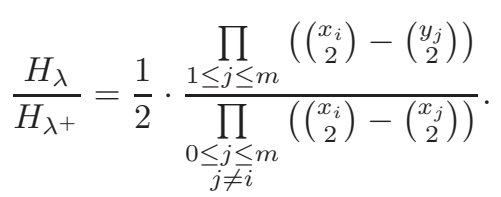

Proof. First we consider the case $i=0$, which means that $y_{1} \geq 2$. In this case we add the box $\square=(\ell+1, \ell+2)$ to $\lambda$ with $c_{\square}=x_{0}=1$ where $\ell$ is the length of $\lambda$. By the definition, it is easy to see that the hook lengths of boxes which are in the $(\ell+1)$-th column and $(\ell+2)$-th column of $\lambda$ increase by 1 , and the hook lengths of boxes in the other columns don't change. Since the boxes which are in the $\ell+1$-column and $\ell+2$-column of $\lambda$ have hook lengths

$$
\bigcup_{j=1}^{m}\left(\left\{h: y_{j}-1 \leq h \leq x_{j}-2\right\} \cup\left\{h: y_{j} \leq h \leq x_{j}-1\right\}\right),
$$

then the boxes which are in the $\ell+1$-column and $\ell+2$-column of $\lambda^{+}$have hook lengths

$$
\{1\} \cup\left(\bigcup_{j=1}^{m}\left(\left\{h: y_{j} \leq h \leq x_{j}-1\right\} \cup\left\{h: y_{j}+1 \leq h \leq x_{j}\right\}\right)\right) .
$$

Therefore

$$
\begin{aligned}
\mathcal{H}(\lambda) \backslash \mathcal{H}\left(\lambda^{+}\right)= & \left\{y_{1}, y_{1}-1, y_{2}, y_{2}-1, \cdots, y_{m}, y_{m}-1\right\} \\
& \backslash\left\{1, x_{1}, x_{1}-1, x_{2}, x_{2}-1, \cdots, x_{m}, x_{m}-1\right\},
\end{aligned}
$$

which means that

$$
\frac{H_{\lambda}}{H_{\lambda^{+}}}=\frac{\prod_{1 \leq j \leq m} y_{j}\left(y_{j}-1\right)}{\prod_{1 \leq j \leq m} x_{j}\left(x_{j}-1\right)}=\frac{\prod_{1 \leq j \leq m}\left(\left(\begin{array}{c}
x_{0} \\
2
\end{array}\right)-\left(\begin{array}{c}
y_{j} \\
2
\end{array}\right)\right)}{\prod_{1 \leq j \leq m}\left(\left(\begin{array}{c}
x_{0} \\
2
\end{array}\right)-\left(\begin{array}{c}
x_{j} \\
2
\end{array}\right)\right)}
$$

since $x_{0}=1$.

Similarly, for the case $1 \leq i \leq m$, we add the box $\square=\left(\alpha_{i+1}+1, \beta_{i}+1\right)$ to $\lambda$ with $c_{\square}=x_{i}$. By the definition, it is easy to see that the hook lengths of boxes which are in the $\left(\alpha_{i+1}+1\right)$-th column, $\left(\beta_{i}+1\right)$-th column and $\left(\alpha_{i+1}+1\right)$-th row of $\lambda$ increase by 1 , and the hook lengths of other boxes don't change. Since the boxes

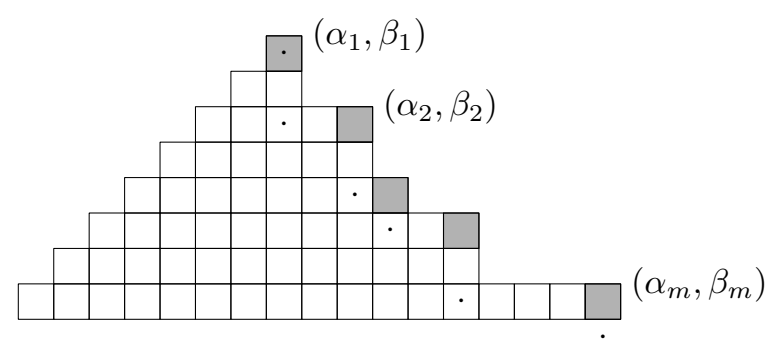

FiguRE 3. A strict partition and its corners. The outer corners are labelled with $\left(\alpha_{i}, \beta_{i}\right)(i=1,2, \ldots, m)$. The inner corners are indicated by the dot symbol ".". 
which are in the $\left(\alpha_{i+1}+1\right)$-th column, $\left(\beta_{i}+1\right)$-th column and $\left(\alpha_{i+1}+1\right)$-th row of $\lambda$ have hook lengths

$$
\begin{gathered}
\bigcup_{j=i+1}^{m}\left\{h: x_{i}+y_{j}-1 \leq h \leq x_{i}+x_{j}-2\right\}, \\
\bigcup_{j=i+1}^{m}\left\{h: y_{j}-x_{i} \leq h \leq x_{j}-x_{i}-1\right\},
\end{gathered}
$$

and

$$
\begin{aligned}
& \left\{h: x_{i}-y_{1} \leq h \leq x_{i}-1\right\} \cup\left\{h: x_{i}+y_{i}-1 \leq h \leq 2 x_{i}-3\right\} \\
& \cup \bigcup_{j=1}^{i-1}\left(\left\{h: x_{i}-y_{j+1} \leq h \leq x_{i}-x_{j}-1\right\} \cup\left\{h: x_{i}+y_{j}-1 \leq h \leq x_{i}+x_{j}-2\right\}\right)
\end{aligned}
$$

respectively, then the same boxes which are in the $\left(\alpha_{i+1}+1\right)$-th column, $\left(\beta_{i}+1\right)$-th column and $\left(\alpha_{i+1}+1\right)$-th row of $\lambda^{+}$have hook lengths

$$
\begin{aligned}
& \bigcup_{j=i+1}^{m}\left\{h: x_{i}+y_{j} \leq h \leq x_{i}+x_{j}-1\right\}, \\
& \bigcup_{j=i+1}^{m}\left\{h: y_{j}-x_{i}+1 \leq h \leq x_{j}-x_{i}\right\},
\end{aligned}
$$

and

$$
\begin{aligned}
& \left\{h: x_{i}-y_{1}+1 \leq h \leq x_{i}\right\} \cup\left\{h: x_{i}+y_{i} \leq h \leq 2 x_{i}-2\right\} \\
& \cup \bigcup_{j=1}^{i-1}\left(\left\{h: x_{i}-y_{j+1}+1 \leq h \leq x_{i}-x_{j}\right\} \cup\left\{h: x_{i}+y_{j} \leq h \leq x_{i}+x_{j}-1\right\}\right)
\end{aligned}
$$

respectively. Notice that the box $\square=\left(\alpha_{i+1}+1, \beta_{i}+1\right)$ in $\lambda^{+}$has hook length 1 , then we have

$$
\begin{gathered}
\mathcal{H}(\lambda) \backslash \mathcal{H}\left(\lambda^{+}\right)=\left(\left\{\left|x_{i}-y_{j}\right|: 1 \leq j \leq m\right\} \cup\left\{x_{i}+y_{j}-1: 1 \leq j \leq m\right\}\right) \\
\backslash\left(\left\{1, x_{i}, 2 x_{i}-2\right\} \cup\left\{\left|x_{i}-x_{j}\right|: 1 \leq j \leq m, j \neq i\right\}\right. \\
\left.\cup\left\{x_{i}+x_{j}-1: 1 \leq j \leq m, j \neq i\right\}\right),
\end{gathered}
$$

which means that

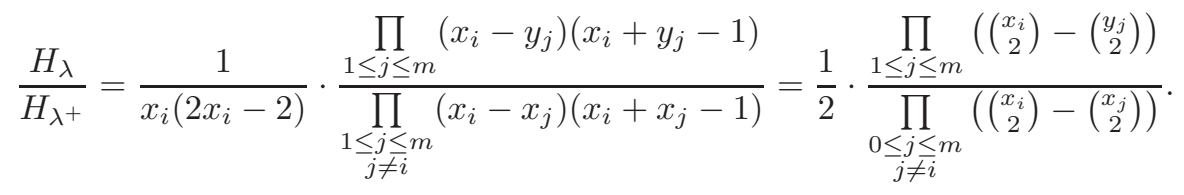

Suppose that $a_{0}<a_{1}<\cdots<a_{m}$ and $b_{1}<\cdots<b_{m}$ are real numbers. Let

$$
q_{k}\left(\left\{a_{i}\right\},\left\{b_{i}\right\}\right):=\sum_{i=0}^{m} a_{i}{ }^{k}-\sum_{i=1}^{m} b_{i}{ }^{k}
$$


for each $k \geq 0$ and

$$
q_{\nu}\left(\left\{a_{i}\right\},\left\{b_{i}\right\}\right):=\prod_{j=1}^{\ell} q_{\nu_{j}}\left(\left\{a_{i}\right\},\left\{b_{i}\right\}\right)
$$

for the usual partition $\nu=\left(\nu_{1}, \nu_{2}, \ldots, \nu_{\ell}\right)$. Notice that

$$
q_{k}(\lambda)=q_{k}\left(\left\{\left(\begin{array}{c}
x_{i} \\
2
\end{array}\right)\right\}_{0 \leq i \leq m},\left\{\left(\begin{array}{c}
y_{i} \\
2
\end{array}\right)\right\}_{1 \leq i \leq m}\right) .
$$

Theorem 3.2. Let $k$ be a nonnegative integer. Then there exist some $\xi_{\nu} \in \mathbb{Q}$ such that

$$
\sum_{0 \leq i \leq m} \frac{\prod_{\substack { 1 \leq j \leq m \\
\begin{subarray}{c}{0 \leq j \leq m \\
j \neq i{ 1 \leq j \leq m \\
\begin{subarray} { c } { 0 \leq j \leq m \\
j \neq i } }\end{subarray}}\left(a_{i}-b_{j}\right)}{\left.\prod_{i}-a_{j}\right)} a_{i}^{k}=\sum_{|\nu| \leq k} \xi_{\nu} q_{\nu}\left(\left\{a_{i}\right\},\left\{b_{i}\right\}\right)
$$

for all real numbers $a_{0}<a_{1}<\cdots<a_{m}$ and $b_{1}<b_{2}<\cdots<b_{m}$.

Proof. First notice we just need to prove the case that $a_{i} \neq 0$ for all $i$. Because if we multiply by $\prod_{0 \leq i<j<n}\left(a_{i}-a_{j}\right)$ on both sides of the above formula, then both sides become polynomials in $a_{0}, a_{1}, \ldots, a_{m}$ and $b_{1}, b_{2}, \ldots, b_{m}$, which means they are continuous functions on such variables. Therefore if the above formula is true for all nonzero $a_{i}$, then it is also true for the case $a_{i}=0$ for some $i$. Let

$$
g(z)=\prod_{1 \leq j \leq m}\left(1-b_{j} z\right)-\sum_{0 \leq i \leq m} \frac{\prod_{\substack{1 \leq j \leq m \\ 0 \leq j \leq m}}\left(a_{i}-b_{j}\right)}{\prod_{\substack{j \neq i \\ 0 .}}\left(a_{i}-a_{j}\right)} \prod_{\substack{0 \leq j \leq m \\ j \neq i}}\left(1-a_{j} z\right) .
$$

Then for $0 \leq i \leq m$ we obtain

$$
g\left(\frac{1}{a_{i}}\right)=\prod_{1 \leq j \leq m}\left(1-\frac{b_{j}}{a_{i}}\right)-\frac{\prod_{\substack{1 \leq j \leq m \\ 0 \leq j \leq m \\ j \neq i}}\left(a_{i}-b_{j}\right)}{\left.\prod_{i}\right)} \prod_{\substack{0 \leq j \leq m \\ j \neq i}}\left(1-\frac{a_{j}}{a_{i}}\right)=0 .
$$

This means that $g(z)$ has at least $m+1$ roots, so that $g(z)=0$ since $g(z)$ is a polynomial in $z$ with degree at most $m$. Therefore

$$
\sum_{0 \leq i \leq m} \frac{\prod_{\substack{1 \leq j \leq m \\ 0 \leq j \leq m \\ j \neq i}}\left(a_{i}-b_{j}\right)}{\prod_{\substack{0 \\ 0}}\left(a_{i}\right)} \cdot \frac{1}{1-a_{i} z}=\frac{\prod_{1 \leq j \leq m}\left(1-b_{j} z\right)}{\prod_{0 \leq j \leq m}\left(1-a_{j} z\right)}
$$

which means that

$$
\begin{aligned}
& \sum_{0 \leq i \leq m} \frac{\prod_{\substack{1 \leq j \leq m \\
0 \leq j \leq m \\
j \neq i}}\left(a_{i}-b_{j}\right)}{\left.\prod_{i}-a_{j}\right)}\left(\sum_{k \geq 0}\left(a_{i} z\right)^{k}\right) \\
& =\exp \left(\sum_{1 \leq j \leq m} \ln \left(1-b_{j} z\right)-\sum_{0 \leq i \leq m} \ln \left(1-a_{i} z\right)\right)
\end{aligned}
$$




$$
=\exp \left(\sum_{k \geq 1} \frac{q_{k}\left(\left\{a_{i}\right\},\left\{b_{i}\right\}\right)}{k} z^{k}\right) .
$$

Comparing the coefficients of $z^{k}$ on both sides, we obtain there exist some $\xi_{\nu} \in \mathbb{Q}$ such that

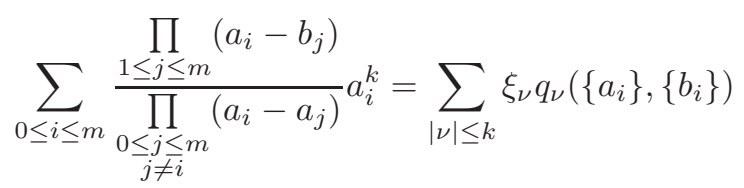

for all real numbers $a_{0}<a_{1}<\cdots<a_{m}$ and $b_{1}<b_{2}<\cdots<b_{m}$.

By (3.6), when $k=0,1,2$, we obtain

$$
\begin{aligned}
& \sum_{0 \leq i \leq m} \frac{\prod_{\substack{1 \leq j \leq m \\
0 \leq j \leq m \\
j \neq i}}\left(a_{i}-b_{j}\right)}{\left.\prod_{i}-a_{j}\right)}=1, \\
& \sum_{0 \leq i \leq m} \frac{\prod_{\substack{1 \leq j \leq m \\
0 \leq j \leq m \\
j \neq i}}\left(a_{i}-b_{j}\right)}{\left.\prod_{i}-a_{j}\right)} a_{i}=q_{1}\left(\left\{a_{i}\right\},\left\{b_{i}\right\}\right), \\
& \sum_{0 \leq i \leq m} \frac{\prod_{\substack{1 \leq j \leq m \\
0 \leq j \leq m}}\left(a_{i}-b_{j}\right)}{\prod_{\substack{0 \\
j \neq i}}\left(a_{i}\right)} a_{i}^{2}=\frac{q_{1}^{2}\left(\left\{a_{i}\right\},\left\{b_{i}\right\}\right)+q_{2}\left(\left\{a_{i}\right\},\left\{b_{i}\right\}\right)}{2} .
\end{aligned}
$$

Let $\lambda^{i+}=\lambda \cup\left\{\square_{i}\right\}$ such that $c_{\square_{i}}=x_{i}$ for $1 \leq i \leq m$. If $y_{1}>1$, let $\lambda^{0+}=\lambda \cup\left\{\square_{0}\right\}$ such that $c_{\square_{0}}=x_{0}=1$.

Theorem 3.3. Suppose that $\lambda$ is a given strict partition. Then

$$
D\left(\frac{1}{H_{\lambda}}\right)=0 .
$$

Proof. Notice that when $y_{1}=1$, we have $\left\{\lambda^{+}: \ell\left(\lambda^{+}\right)>\ell(\lambda)\right\}=\emptyset$, therefore

$$
\sum_{\ell\left(\lambda^{+}\right)>\ell(\lambda)} \frac{H_{\lambda}}{H_{\lambda^{+}}}=0=\frac{\prod_{1 \leq j \leq m}\left(\left(\begin{array}{c}
x_{0} \\
2
\end{array}\right)-\left(\begin{array}{c}
y_{j} \\
2
\end{array}\right)\right)}{\prod_{1 \leq j \leq m}\left(\left(\begin{array}{c}
x_{0} \\
2
\end{array}\right)-\left(\begin{array}{c}
x_{j} \\
2
\end{array}\right)\right)} .
$$

When $y_{1}>1$, we have $\left\{\lambda^{+}: \ell\left(\lambda^{+}\right)>\ell(\lambda)\right\}=\left\{\lambda^{0+}\right\}$, therefore by Theorem 3.1 we also obtain

$$
\sum_{\ell\left(\lambda^{+}\right)>\ell(\lambda)} \frac{H_{\lambda}}{H_{\lambda^{+}}}=\frac{\prod_{1 \leq j \leq m}\left(\left(\begin{array}{c}
x_{0} \\
2
\end{array}\right)-\left(\begin{array}{c}
y_{j} \\
2
\end{array}\right)\right)}{\prod_{1 \leq j \leq m}\left(\left(\begin{array}{c}
x_{0} \\
2
\end{array}\right)-\left(\begin{array}{c}
x_{j} \\
2
\end{array}\right)\right)}
$$

and

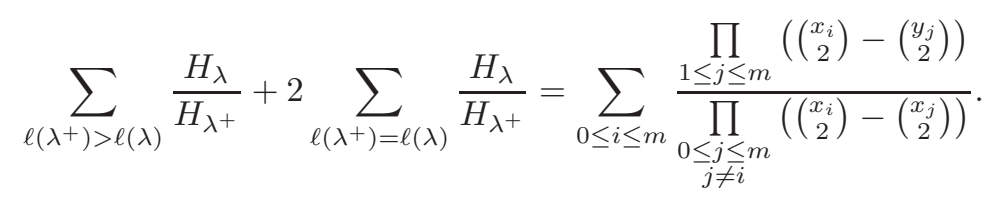


Let $a_{i}=\left(\begin{array}{c}x_{i} \\ 2\end{array}\right)$ and $b_{i}=\left(\begin{array}{c}y_{i} \\ 2\end{array}\right)$ in (3.7), we obtain

$$
D\left(\frac{1}{H_{\lambda}}\right)=\frac{1}{H_{\lambda}}\left(\sum_{\ell\left(\lambda^{+}\right)>\ell(\lambda)} \frac{H_{\lambda}}{H_{\lambda^{+}}}+2 \sum_{\ell\left(\lambda^{+}\right)=\ell(\lambda)} \frac{H_{\lambda}}{H_{\lambda^{+}}}-1\right)=0 .
$$

Corollary 3.4. Suppose that $g$ is a function on strict partitions. Then

$$
D\left(\frac{g(\lambda)}{H_{\lambda}}\right)=\sum_{\ell\left(\lambda^{+}\right)>\ell(\lambda)} \frac{g\left(\lambda^{+}\right)-g(\lambda)}{H_{\lambda^{+}}}+2 \sum_{\ell\left(\lambda^{+}\right)=\ell(\lambda)} \frac{g\left(\lambda^{+}\right)-g(\lambda)}{H_{\lambda^{+}}}
$$

for every strict partition $\lambda$.

Proof. The corollary follows directly from the definition of the operator $D$ and the last identity in the proof of Theorem 3.3 .

Theorem 3.5. Let $k$ be a given nonnegative integer and $\lambda$ be a strict partition. Then there exist some $\xi_{j} \in \mathbb{Q}$ such that

$$
q_{k}\left(\lambda^{i+}\right)-q_{k}(\lambda)=\sum_{j=0}^{k-1} \xi_{j}\left(\begin{array}{c}
x_{i} \\
2
\end{array}\right)^{j}
$$

for every strict partition $\lambda$ and every $i$.

Proof. Denote by $X=\left\{x_{0}, x_{1}, \ldots, x_{m}\right\}$ and $Y=\left\{y_{1}, y_{2}, \ldots, y_{m}\right\}$. For $1 \leq i \leq m$, four cases are to be considered.

(i) If $\beta_{i}+1<\beta_{i+1}$ and $\alpha_{i+1}+1<\alpha_{i}$. Then it is easy to see that the contents of inner corners and outer corners of $\lambda^{i+}$ are $X \cup\left\{x_{i}-1, x_{i}+1\right\} \backslash\left\{x_{i}\right\}$ and $Y \cup\left\{x_{i}\right\}$ respectively.

(ii) If $\beta_{i}+1=\beta_{i+1}$ and $\alpha_{i+1}+1<\alpha_{i}$, so that $y_{i+1}=x_{i}+1$. Hence the contents of inner corners and outer corners of $\lambda^{i+}$ are $X \cup\left\{x_{i}-1\right\} \backslash\left\{x_{i}\right\}$ and $Y \cup\left\{x_{i}\right\} \backslash\left\{x_{i}+1\right\}$ respectively.

(iii) If $\beta_{i}+1<\beta_{i+1}$ and $\alpha_{i+1}+1=\alpha_{i}$, so that $y_{i}=x_{i}-1$. Then the contents of inner corners and outer corners of $\lambda^{i+}$ are $X \cup\left\{x_{i}+1\right\} \backslash\left\{x_{i}\right\}$ and $Y \cup\left\{x_{i}\right\} \backslash\left\{x_{i}-1\right\}$ respectively.

(iv) If $\beta_{i}+1=\beta_{i+1}$ and $\alpha_{i+1}+1=\alpha_{i}$. Then $y_{i}+1=x_{i}=y_{i+1}-1$. The contents of inner corners and outer corners of $\lambda^{i+}$ are $X \backslash\left\{x_{i}\right\}$ and $Y \cup\left\{x_{i}\right\} \backslash\left\{x_{i}-1, x_{i}+1\right\}$ respectively.

For $i=0$, two cases are to be considered.

(v) If $y_{1}=2$, the contents of inner corners and outer corners of $\lambda^{0+}$ are $X$ and $Y \cup\{1\} \backslash\{2\}$ respectively.

(vi) If $y_{1}>2$, the contents of inner corners and outer corners of $\lambda^{i+}$ are $X \cup\{2\}$ and $Y \cup\{1\}$ respectively.

In each of the six cases, we always have

$$
q_{k}\left(\lambda^{i+}\right)-q_{k}(\lambda)=\left(\begin{array}{c}
x_{i}+1 \\
2
\end{array}\right)^{k}+\left(\begin{array}{c}
x_{i}-1 \\
2
\end{array}\right)^{k}-2\left(\begin{array}{c}
x_{i} \\
2
\end{array}\right)^{k} .
$$

Next we have for all $z \in \mathbb{R}$,

$$
(z+2)^{2 k}+(z-2)^{2 k}-2 z^{2 k}=2 \sum_{1 \leq j \leq k}\left(\begin{array}{l}
2 k \\
2 j
\end{array}\right) 2^{2 j} z^{2 k-2 j} .
$$


Replace $z$ by $2 z-1$, we obtain

$$
(2 z+1)^{2 k}+(2 z-3)^{2 k}-2(2 z-1)^{2 k}=2 \sum_{1 \leq j \leq k}\left(\begin{array}{c}
2 k \\
2 j
\end{array}\right) 2^{2 j}(2 z-1)^{2 k-2 j}
$$

or

$$
\begin{aligned}
& \left(8\left(\begin{array}{c}
z+1 \\
2
\end{array}\right)+1\right)^{k}+\left(8\left(\begin{array}{c}
z-1 \\
2
\end{array}\right)+1\right)^{k}-2\left(8\left(\begin{array}{l}
z \\
2
\end{array}\right)+1\right)^{k} \\
= & 2 \sum_{1 \leq j \leq k}\left(\begin{array}{c}
2 k \\
2 j
\end{array}\right) 2^{2 j}\left(8\left(\begin{array}{l}
z \\
2
\end{array}\right)+1\right)^{k-j} .
\end{aligned}
$$

Then by induction on $k$ we have

$$
\left(\begin{array}{c}
x_{i}+1 \\
2
\end{array}\right)^{k}+\left(\begin{array}{c}
x_{i}-1 \\
2
\end{array}\right)^{k}-2\left(\begin{array}{c}
x_{i} \\
2
\end{array}\right)^{k}=\sum_{j=0}^{k-1} \xi_{j}\left(\begin{array}{c}
x_{i} \\
2
\end{array}\right)^{j}
$$

for some constants $\xi_{j} \in \mathbb{Q}$.

Theorem 3.6. Let $\nu=\left(\nu_{1}, \nu_{2}, \ldots, \nu_{\ell}\right)$ be a partition. Then there exist some $\xi_{\delta} \in \mathbb{Q}$ such that

$$
D\left(\frac{q_{\nu}(\lambda)}{H_{\lambda}}\right)=\sum_{|\delta| \leq|\nu|-1} \xi_{\delta} \frac{q_{\delta}(\lambda)}{H_{\lambda}}
$$

for every strict partition $\lambda$.

Proof. For $0 \leq i \leq m$, we have

$$
\prod_{k=1}^{\ell} q_{\nu_{k}}\left(\lambda^{i+}\right)-\prod_{k=1}^{\ell} q_{\nu_{k}}(\lambda)=\sum_{(*)} \prod_{k \in U} q_{\nu_{k}}(\lambda) \prod_{k^{\prime} \in V}\left(q_{\nu_{k^{\prime}}}\left(\lambda^{i+}\right)-q_{\nu_{k^{\prime}}}(\lambda)\right),
$$

where the sum $(*)$ ranges over all pairs $(U, V)$ of positive integer sets such that $U \cup V=\{1,2, \ldots, \ell\}, U \cap V=\emptyset$ and $V \neq \emptyset$. Actually the Identity (3.12) follows by the inclusion-exclusion principle. By Corollary 3.4 and Theorem 3.1 we have

$$
\begin{aligned}
& H_{\lambda} D\left(\frac{q_{\nu}(\lambda)}{H_{\lambda}}\right)=\sum_{\ell\left(\lambda^{+}\right)>\ell(\lambda)} \frac{H_{\lambda}\left(q_{\nu}\left(\lambda^{+}\right)-q_{\nu}(\lambda)\right)}{H_{\lambda^{+}}}+2 \sum_{\ell\left(\lambda^{+}\right)=\ell(\lambda)} \frac{H_{\lambda}\left(q_{\nu}\left(\lambda^{+}\right)-q_{\nu}(\lambda)\right)}{H_{\lambda^{+}}} \\
& =\sum_{0 \leq i \leq m} \frac{\prod_{\substack{1 \leq j \leq m \\
0 \leq j \leq m \\
j \neq i}}\left(\left(\begin{array}{c}
x_{i} \\
2
\end{array}\right)-\left(\begin{array}{c}
y_{j} \\
2
\end{array}\right)\right)}{\left.\left.\prod_{0}^{x_{i}}\right)-\left(\begin{array}{c}
x_{j} \\
2
\end{array}\right)\right)}\left(\prod_{k=1}^{\ell} q_{\nu_{k}}\left(\lambda^{i+}\right)-\prod_{k=1}^{\ell} q_{\nu_{k}}(\lambda)\right)
\end{aligned}
$$

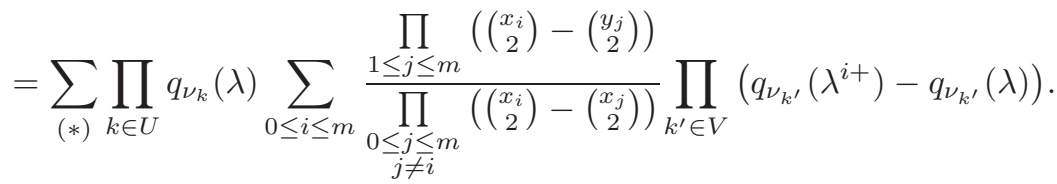

Then the claim follows from Theorems 3.5 and 3.2 , 


\section{Proofs of Theorems}

Instead of proving Theorem 1.1, we prove the following more general result, which implies Theorem 1.1 when $\nu=\emptyset$.

Theorem 4.1. Suppose that $\nu=\left(\nu_{1}, \nu_{2}, \ldots, \nu_{\ell}\right)$ is a given partition, $\mu$ is a given strict partition and $Q$ is a symmetric function. Then there exists some $r \in \mathbb{N}$ such that

$$
D^{r}\left(\frac{Q\left(\left(\begin{array}{c}
c \square \\
2
\end{array}\right): \square \in \lambda\right) q_{\nu}(\lambda)}{H_{\lambda}}\right)=0
$$

for every strict partition $\lambda$. Consequently,

$$
P(n)=\sum_{|\lambda / \mu|=n} \frac{2^{|\lambda|-|\mu|-\ell(\lambda)+\ell(\mu)} f_{\lambda / \mu}}{H_{\lambda}} Q\left(\left(\begin{array}{c}
c_{\square} \\
2
\end{array}\right): \square \in \lambda\right) q_{\nu}(\lambda)
$$

is a polynomial in $n$.

Proof. By linearity we can assume that

$$
Q\left(\left(\begin{array}{c}
c_{\square} \\
2
\end{array}\right): \square \in \lambda\right)=\prod_{t=1}^{s} \sum_{\square \in \lambda}\left(\begin{array}{c}
c_{\square} \\
2
\end{array}\right)^{r_{t}}
$$

for some tuple $\left(r_{1}, r_{2}, \ldots, r_{s}\right)$. Let

$$
\begin{aligned}
A & =q_{\nu}(\lambda), \\
\Delta_{i} A & =q_{\nu}\left(\lambda^{i+}\right)-q_{\nu}(\lambda), \\
B & =\prod_{t=1}^{s} \sum_{\square \in \lambda}\left(\begin{array}{c}
c_{\square} \\
2
\end{array}\right)^{r_{t}}, \\
\Delta_{i} B & =\prod_{t=1}^{s} \sum_{\square \in \lambda^{i+}}\left(\begin{array}{c}
c_{\square} \\
2
\end{array}\right)^{r_{t}}-\prod_{t=1}^{s} \sum_{\square \in \lambda}\left(\begin{array}{c}
c_{\square} \\
2
\end{array}\right)^{r_{t}} .
\end{aligned}
$$

We have

$$
\begin{aligned}
\Delta_{i} A & =\sum_{(*)} \prod_{k \in U} q_{\nu_{k}}(\lambda) \prod_{k^{\prime} \in V}\left(q_{\nu_{k^{\prime}}}\left(\lambda^{i+}\right)-q_{\nu_{k^{\prime}}}(\lambda)\right), \\
\Delta_{i} B & =\sum_{(* *)} \prod_{t \in U} \sum_{\square \in \lambda}\left(\begin{array}{c}
c_{\square} \\
2
\end{array}\right)^{r_{t}} \prod_{t^{\prime} \in V}\left(\sum_{\square \in \lambda^{i+}}\left(\begin{array}{c}
c_{\square} \\
2
\end{array}\right)^{r_{t^{\prime}}}-\sum_{\square \in \lambda}\left(\begin{array}{c}
c_{\square} \\
2
\end{array}\right)^{r_{t^{\prime}}}\right) \\
& =\sum_{(* *)} \prod_{t \in U} \sum_{\square \in \lambda}\left(\begin{array}{c}
c_{\square} \\
2
\end{array}\right)^{r_{t}} \prod_{t^{\prime} \in V}\left(\begin{array}{c}
x_{i} \\
2
\end{array}\right)^{r_{t^{\prime}}},
\end{aligned}
$$

where the sum $(*)$ (resp. $(* *))$ ranges over all pairs $(U, V)$ of positive integer sets such that $U \cup V=\{1,2, \ldots, \ell\}$ (resp. $U \cup V=\{1,2, \ldots, s\}), U \cap V=\emptyset$ and $V \neq \emptyset$. It follows from Corollary 3.4 and Theorem 3.1 that

$$
\begin{aligned}
& H_{\lambda} D\left(\frac{q_{\nu}(\lambda) \prod_{t=1}^{s} \sum_{\square \in \lambda}\left(\begin{array}{c}
c_{\square} \\
2
\end{array}\right)^{r_{t}}}{H_{\lambda}}\right) \\
= & \sum_{\ell\left(\lambda^{+}\right)>\ell(\lambda)} \frac{H_{\lambda}}{H_{\lambda^{+}}}\left(q_{\nu}\left(\lambda^{+}\right) \prod_{t=1}^{s} \sum_{\square \in \lambda^{+}}\left(\begin{array}{c}
c_{\square} \\
2
\end{array}\right)^{r_{t}}-q_{\nu}(\lambda) \prod_{t=1}^{s} \sum_{\square \in \lambda}\left(\begin{array}{c}
c_{\square} \\
2
\end{array}\right)^{r_{t}}\right)
\end{aligned}
$$




$$
\begin{aligned}
& +2 \sum_{\ell\left(\lambda^{+}\right)=\ell(\lambda)} \frac{H_{\lambda}}{H_{\lambda^{+}}}\left(q_{\nu}\left(\lambda^{+}\right) \prod_{t=1}^{s} \sum_{\square \in \lambda^{+}}\left(\begin{array}{c}
c_{\square} \\
2
\end{array}\right)^{r_{t}}-q_{\nu}(\lambda) \prod_{t=1}^{s} \sum_{\square \in \lambda}\left(\begin{array}{c}
c_{\square} \\
2
\end{array}\right)^{r_{t}}\right) \\
& =\sum_{i=0}^{m} \frac{\left.\prod_{\substack{1 \leq j \leq m \\
0 \leq j \leq m \\
j \neq i}}\left(\left(\begin{array}{c}
x_{i} \\
2
\end{array}\right)-\left(\begin{array}{c}
y_{j} \\
2
\end{array}\right)\right)-\left(\begin{array}{c}
x_{j} \\
2
\end{array}\right)\right)}{\prod_{j}}\left(q_{\nu}\left(\lambda^{i+}\right) \prod_{t=1}^{s} \sum_{\square \in \lambda^{i+}}\left(\begin{array}{c}
c_{\square} \\
2
\end{array}\right)^{r_{t}}-q_{\nu}(\lambda) \prod_{t=1}^{s} \sum_{\square \in \lambda}\left(\begin{array}{c}
c_{\square} \\
2
\end{array}\right)^{r_{t}}\right)
\end{aligned}
$$

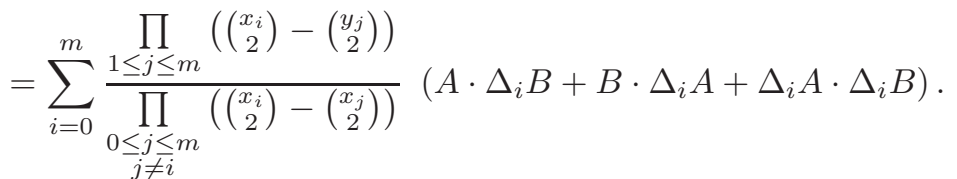

Then by Theorems 3.2, 3.5 and 3.6 each of the above three terms could be written as a linear combination of some $\prod_{\bar{t}=1}^{\bar{s}} \sum_{\square \in \lambda}\left(\begin{array}{c}c_{\square} \\ 2\end{array}\right)^{\bar{r}_{\bar{t}}} q_{\bar{\nu}}(\lambda)$ satisfying one of the following two conditions:

(1) $\bar{s}<s$;

(2) $\bar{s}=s$ and $|\bar{\nu}| \leq|\nu|-1$.

Therefore the theorem follows by induction on $s$ and $|\nu|$.

Proof of Theorem 1.2. The special case in the proof of Theorem 4.1 with $\nu=\emptyset$ and $s=1$ yields

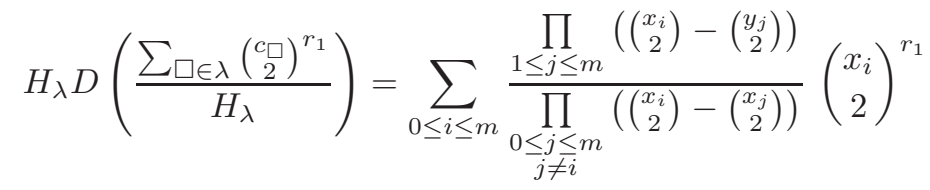

$$
\begin{aligned}
& =\sum_{|\nu| \leq r_{1}} \xi_{\nu} q_{\nu}(\lambda),
\end{aligned}
$$

where $\xi_{\nu}$ are some constants. The last equality is due to Theorem 3.2 . Notice that

$$
(2 k) !\left(\begin{array}{c}
z+k-1 \\
2 k
\end{array}\right)=2^{k} \prod_{i=1}^{k}\left(\left(\begin{array}{l}
z \\
2
\end{array}\right)-\left(\begin{array}{l}
i \\
2
\end{array}\right)\right) .
$$

Then by Theorems 3.6 and 2.3 we know that

$$
P(n)=\sum_{|\lambda|=n} \frac{f_{\lambda}^{\prime}}{H_{\lambda}} \sum_{\square \in \lambda}\left(\begin{array}{c}
c_{\square}+k-1 \\
2 k
\end{array}\right)
$$

is a polynomial in $n$ with degree at most $k+1$.

On the other hand,

$$
P(k+1)=\frac{f^{\prime}(k+1)}{H_{(k+1)}}\left(\begin{array}{l}
2 k \\
2 k
\end{array}\right)=\frac{2^{k}}{(k+1) !}
$$

since $\lambda=(k+1)$ is the only strict partition with size $k+1$ who has contents greater than $k$. Moreover, it is obvious that $P(0)=P(1)=\cdots=P(k)=0$. Since the polynomial $P(n)$ is uniquely determined by those values, we obtain

$$
P(n)=\frac{2^{k}}{(k+1) !}\left(\begin{array}{c}
n \\
k+1
\end{array}\right) .
$$


By Theorem 4.1, the left-hand side of (1.3) is a polynomial in $n$. To evaluate this polynomial explicitly, we need the following lemma.

Lemma 4.2. Let $\lambda$ be a strict partition. Then

$$
q_{1}(\lambda)=|\lambda| .
$$

Proof. By the definition of the size of $\lambda$, we have

$$
|\lambda|=\sum_{i=1}^{m} \sum_{j=y_{i}}^{x_{i}-1} j=\sum_{i=1}^{m}\left(\left(\begin{array}{c}
x_{i} \\
2
\end{array}\right)-\left(\begin{array}{c}
y_{i} \\
2
\end{array}\right)\right)=q_{1}(\lambda) .
$$

Proof of Theorem 1.3. It is easy to check that both sides of (1.3) are equal for $n=0,1,2$. By Corollary 3.4. Theorem 3.1 and Identity (3.8) it is easy to see that

$$
\begin{aligned}
& H_{\lambda} D\left(\frac{\sum_{\square \in \lambda}\left(\begin{array}{c}
c \square \\
2
\end{array}\right)}{H_{\lambda}}\right)=\sum_{\ell\left(\lambda^{+}\right)>\ell(\lambda)} \frac{H_{\lambda}}{H_{\lambda^{+}}}\left(\sum_{\square \in \lambda^{+}}\left(\begin{array}{c}
c \square \\
2
\end{array}\right)-\sum_{\square \in \lambda}\left(\begin{array}{c}
c_{\square} \\
2
\end{array}\right)\right) \\
& +2 \sum_{\ell\left(\lambda^{+}\right)=\ell(\lambda)} \frac{H_{\lambda}}{H_{\lambda^{+}}}\left(\sum_{\square \in \lambda^{+}}\left(\begin{array}{c}
c_{\square} \\
2
\end{array}\right)-\sum_{\square \in \lambda}\left(\begin{array}{c}
c_{\square} \\
2
\end{array}\right)\right) \\
& =\sum_{0 \leq i \leq m} \frac{\prod_{\substack{1 \leq j \leq m \\
0 \leq j \leq m \\
j \neq i}}\left(\left(\begin{array}{c}
x_{i} \\
2
\end{array}\right)-\left(\begin{array}{c}
y_{j} \\
2
\end{array}\right)\right)}{\left.\left.\prod_{0}\right)-\left(\begin{array}{c}
x_{j} \\
2
\end{array}\right)\right)}\left(\begin{array}{c}
x_{i} \\
2
\end{array}\right) \\
& =q_{1}(\lambda) \\
& =|\lambda| \text {. }
\end{aligned}
$$

Therefore we have

$$
\begin{aligned}
& H_{\lambda} D^{2}\left(\frac{\sum_{\square \in \lambda}\left(\begin{array}{c}
c_{\square} \\
2
\end{array}\right)}{H_{\lambda}}\right)=1, \\
& H_{\lambda} D^{3}\left(\frac{\sum_{\square \in \lambda}\left(\begin{array}{c}
c \square \\
2
\end{array}\right)}{H_{\lambda}}\right)=0 .
\end{aligned}
$$

Then our claim follows from Theorem 2.3 .

Similarly, by (3.7), (3.8) and (3.9) we have

$$
\begin{aligned}
& H_{\lambda} D\left(\frac{\sum_{\square \in \lambda}\left({ }_{\square}^{\square_{4}+1}\right)}{H_{\lambda}}\right)=\frac{1}{12}\left(q_{2}(\lambda)+|\lambda|^{2}-2|\lambda|\right), \\
& H_{\lambda} D^{2}\left(\frac{\sum_{\square \in \lambda}\left({ }_{\square}^{\square_{4}+1}\right)}{H_{\lambda}}\right)=\frac{2}{3}|\lambda|, \\
& H_{\lambda} D^{3}\left(\frac{\sum_{\square \in \lambda}\left({ }^{c_{\square}+1}\right)}{H_{\lambda}}\right)=\frac{2}{3}, \\
& H_{\lambda} D^{4}\left(\frac{\sum_{\square \in \lambda}\left({ }^{c_{4}+1}\right)}{H_{\lambda}}\right)=0 .
\end{aligned}
$$


Thus by Theorem 2.3 we obtain the following result.

Theorem 4.3. Let $\mu$ be a strict partition. Then

$$
\begin{aligned}
& \sum_{|\lambda / \mu|=n} \frac{2^{|\lambda|-\ell(\lambda)-|\mu|+\ell(\mu)} f_{\lambda / \mu} H_{\mu}}{H_{\lambda}}\left(\sum_{\square \in \lambda}\left(\begin{array}{c}
c_{\square}+1 \\
4
\end{array}\right)-\sum_{\square \in \mu}\left(\begin{array}{c}
c_{\square}+1 \\
4
\end{array}\right)\right) \\
= & \frac{2}{3}\left(\begin{array}{c}
n \\
3
\end{array}\right)+\frac{2}{3}|\mu|\left(\begin{array}{c}
n \\
2
\end{array}\right)+\frac{1}{12}\left(q_{2}(\mu)+|\mu|^{2}-2|\mu|\right) n .
\end{aligned}
$$

\section{Acknowledgments}

The authors thank the referees for carefully reading the paper and for giving helpful comments. The second author thanks Prof. P.-O. Dehaye for the encouragements and helpful suggestions. The second author is supported by Grant [PP00P2_138906] of the Swiss National Science Foundation.

\section{REFERENCES}

[1] J. Bandlow, An elementary proof of the hook formula, Electron. J. Comb. 15 (2008), research paper 45.

[2] P.-O. Dehaye, G.-N. Han, and H. Xiong, Difference operators for partitions under the Littlewood decomposition, Ramanujan J., to appear.

[3] G.-N. Han, The Nekrasov-Okounkov hook length formula: refinement, elementary proof, extension, and applications, Ann. Inst. Fourier 60(1) (2010), 1-29.

[4] G.-N. Han, Some conjectures and open problems on partition hook lengths, Exp. Math. 18 (2009), 97-106.

[5] G.-N. Han, Hook lengths and shifted parts of partitions, Ramanujan J. 23(1-3) (2010), 127-135.

[6] G.-N. Han and Kathy Q. Ji, Combining hook length formulas and BG-ranks for partitions via the Littlewood decomposition, Trans. Am. Math. Soc. 363 (2011), 1041-1060.

[7] G.-N. Han and H. Xiong, Difference operators for partitions and some applications, preprint; arXiv:1508.00772.

[8] G.-N. Han and H. Xiong, Polynomiality of some hook-content summations for doubled distinct and self-conjugate partitions, preprint; arXiv:1601.04369,

[9] D. Knuth, The Art of Computer Programming, Vol. 3: Sorting and Searching, Addison-Wesley, London, 1973, pp. 54-58.

[10] N. A. Nekrasov and A. Okounkov, Seiberg-Witten theory and random partitions, The unity of mathematics, Progress in Mathematics 244, Birkhäuser Boston, 2006, pp. 525-596.

[11] G. Olshanski, Anisotropic Young diagrams and infinite-dimensional diffusion processes with the Jack parameter, Int. Math. Res. Not. 6 (2010), 1102-1166.

[12] G. Olshanski, Plancherel averages: Remarks on a paper by Stanley, Electron. J. Comb. 17 (2010), research paper 43.

[13] G. Panova, Polynomiality of some hook-length statistics, Ramanujan J. 27(3) (2012), 349-356. 
[14] Rota, Gian-Carlo, On the foundations of combinatorial theory: I. Theory of Möbius functions, Z. Wahrscheinlichkeitstheorie und Verw. Gebiete 2 (1964), 349-356.

[15] I. Schur, Über die Darstellung der symmetrischen und der alternienden Gruppe durch gebrochene lineare Substitutionen, J. Reine Angew. Math. 139 (1911), $155-250$.

[16] R. P. Stanley. Some combinatorial properties of hook lengths, contents, and parts of partitions, Ramanujan J. 23(1-3) (2010), 91-105.

[17] R. P. Stanley, Enumerative Combinatorics, vol. 2, Cambridge University Press, Cambridge, 1999.

[18] R. M. Thrall, A combinatorial problem, Mich. Math. J. 1 (1952), 81-88.

Université de Strasbourg, CNRS, IRMA UMR 7501, F-67000 Strasbourg, France

E-mail address: guoniu.han@unistra.fr, xiong@math.unistra.fr 